\title{
Broad-Spectrum Ultrathin-Metal-Based Oxide/Metal/Oxide Transparent Conductive Films for Optoelectronic Devices
}

Zhang Liu ${ }^{+, 1}$, Yalu Zou ${ }^{+, 2}$, Chengang Ji ${ }^{+, 3}$, Xinliang Chen ${ }^{*, 1}$, Guofu Hou ${ }^{1}$, Cong Zhang ${ }^{1}$, Xiangjian Wan $^{2}$, L. Jay Guo ${ }^{3}$, Ying Zhao ${ }^{1}$, Xiaodan Zhang ${ }^{1}$

${ }^{1}$ Institute of Photo-electronic Thin Film Devices and Technology, Key Laboratory of Photo-electronic Thin Film Devices and Technology of Tianjin, College of Electronic Information and Optical Engineering, Nankai University, Tianjin 300350, China

${ }^{2}$ Key Laboratory of Functional Polymer Materials, Renewable Energy Conversion and Storage Center (RECAST), College of Chemistry, Nankai University, Tianjin 300071, China

${ }^{3}$ Department of Electrical Engineering and Computer Science, University of Michigan, Ann Arbor, Michigan 48109, United States

Keywords: broad-spectrum, transparent conductive films, ultrathin metal film, oxide/metal/oxide, optoelectronic devices

${ }^{+}$Z. Liu, Y.L. Zou, C.G. Ji contributed equally to this work.

*Corresponding Author. E-mail: cxlruzhou@163.com (X.L. Chen) 
I. Comparison of the properties of Ag-Zn thin films on different substrates (including the optical, electrical and structural performances).
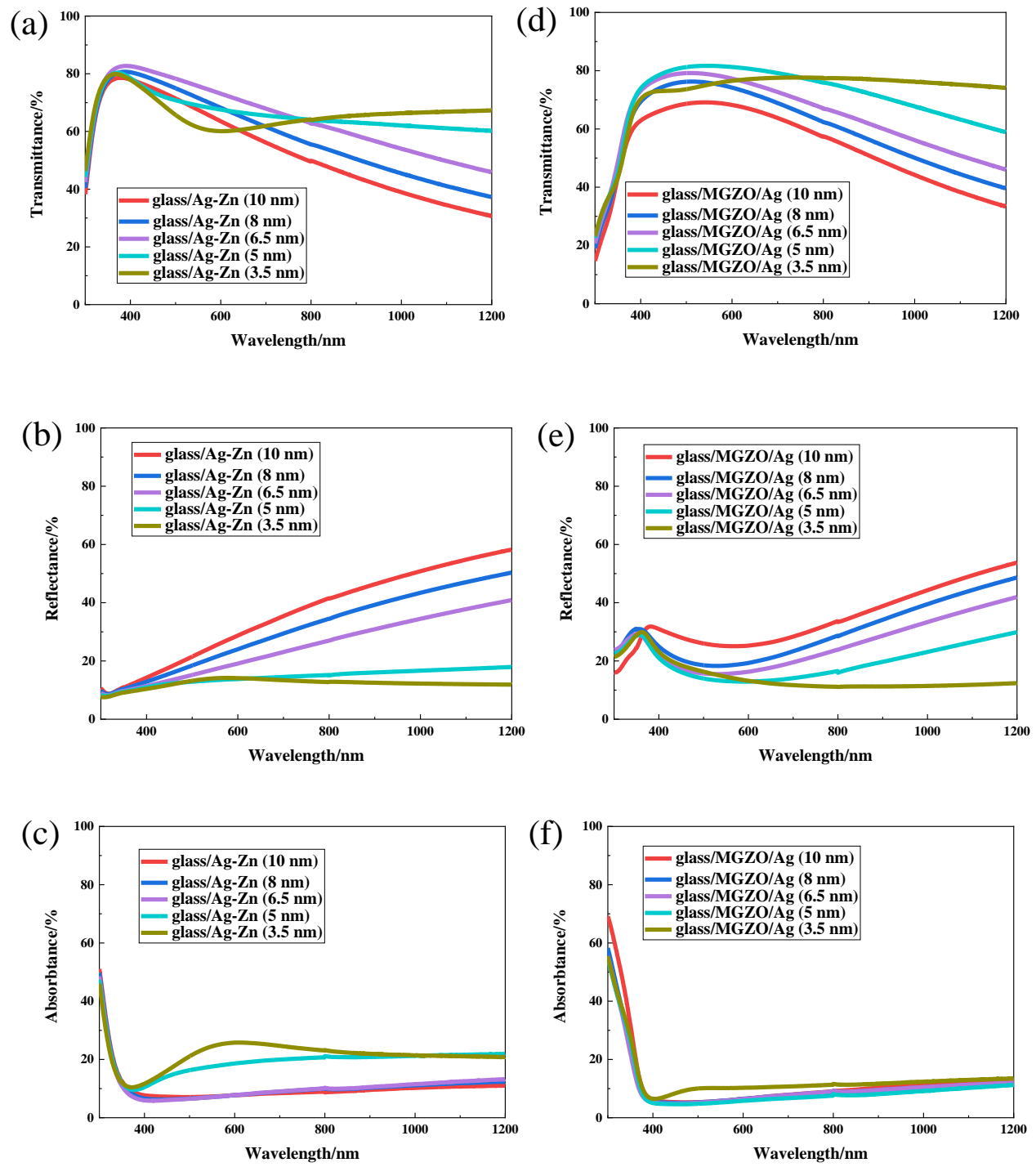

Figure S1. (a) Transmittance, (b) reflectance, (c) absorbance of glass/Ag-Zn thin films, and (d) transmittance, (e) reflectance, (f) absorbance of glass/MGZOAg-Zn thin films with different thickness of Ag-Zn films. It can be observed that the turning point of transmittance and absorbance is $6.5 \mathrm{~nm}$ on glass substrate and $5 \mathrm{~nm}$ on glass/MGZO substrate, respectively. 

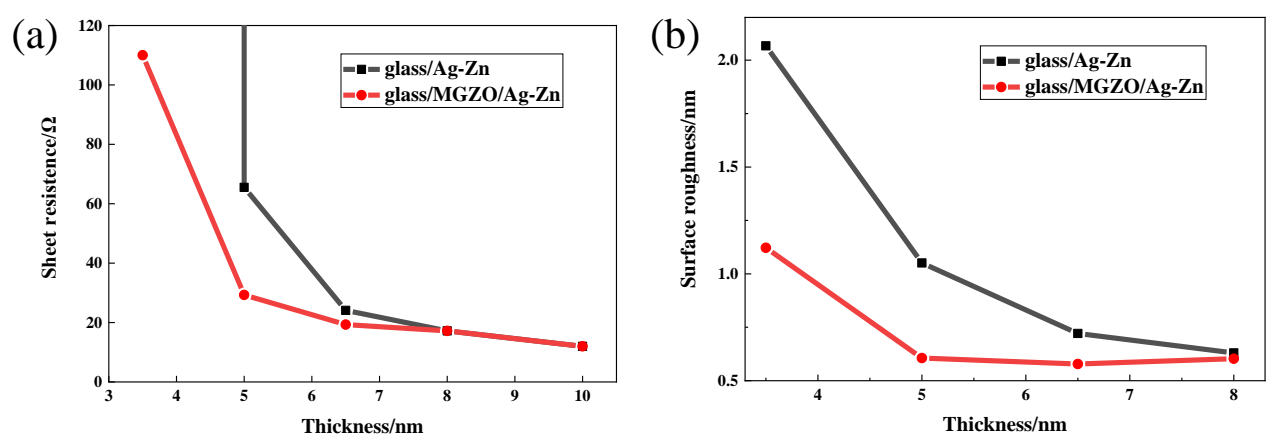

Figure S2. (a) Sheet resistance and (b) surface roughness of glass/Ag-Zn and glass/MGZO/Ag-Zn with different thicknesses of Ag-Zn films. The falling speed of sheet resistance and surface roughness of $\mathrm{Ag}-\mathrm{Zn}$ films on glass/MGZO substrate is significantly faster than that on glass substrate, which indicates MGZO can promote the nucleation of Ag-Zn thin films. 

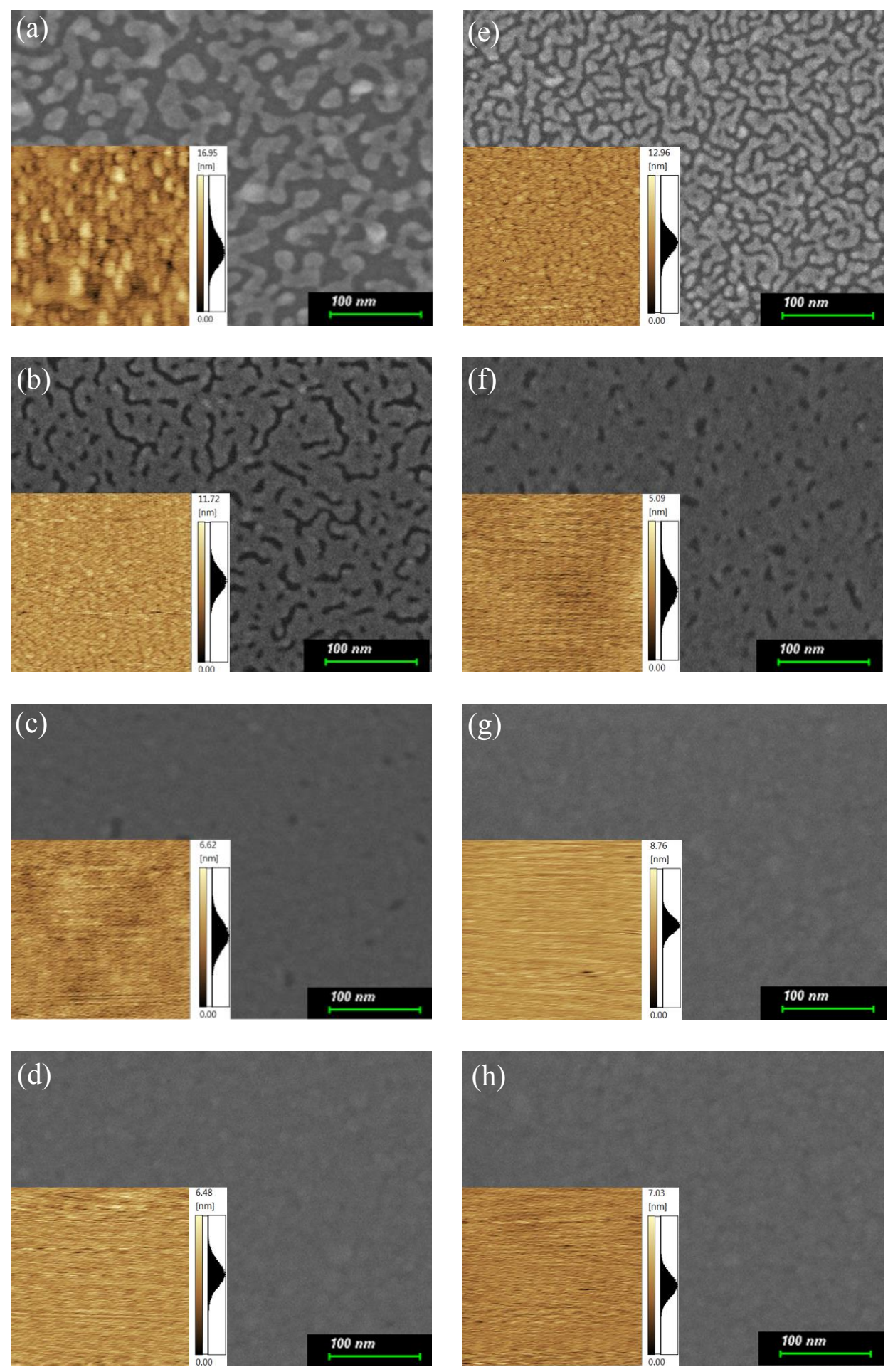

Figure S3. The surface morphology of glass/Ag-Zn with different thicknesses of Ag-Zn films: (a) 3.5 nm; (b) $5 \mathrm{~nm}$; (c) $6.5 \mathrm{~nm}$; (d) $8 \mathrm{~nm}$; The surface morphology of glass/MGZO/Ag-Zn with different thickness of Ag-Zn films: (e) $3.5 \mathrm{~nm}$; (f) $5 \mathrm{~nm}$; (g) $6.5 \mathrm{~nm}$; (h) $8 \mathrm{~nm}$ (The scanning range of AFM is $500 \times 500 \mathrm{~nm}^{2}$ ). The thickness of the Ag-Zn film continuously formed on the glass substrate and the glass/MGZO substrate (the threshold thickness) is $6.5 \mathrm{~nm}$ and $5 \mathrm{~nm}$, respectively. 


\section{Optical, electrical and structural performances of Ag-Al thin films.}

(a)

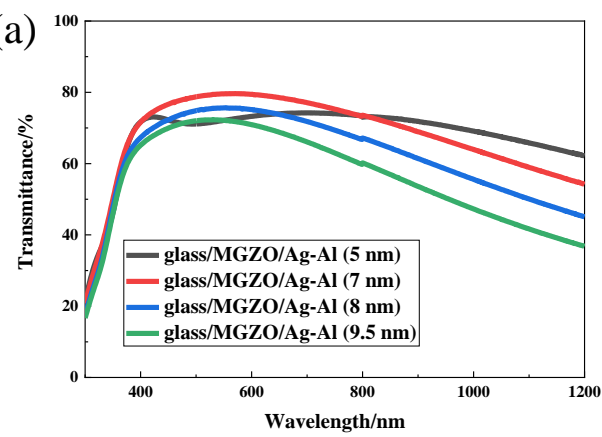

(b)

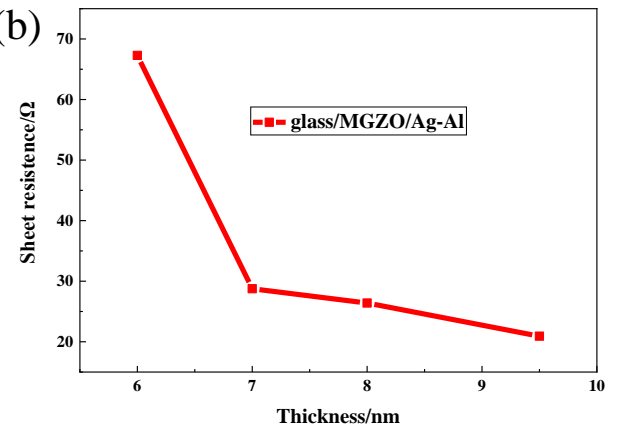

Figure S4. (a) Transmittance and (b) sheet resistance of glass/MGZO/Ag-Al with different thicknesses of Ag-Al films. According to the turning point of transmittance and the sudden increase point of sheet resistance, it can be considered that the threshold thickness of Ag-Al thin film is $7 \mathrm{~nm}$ on the glass/MGZO substrate.

(a)

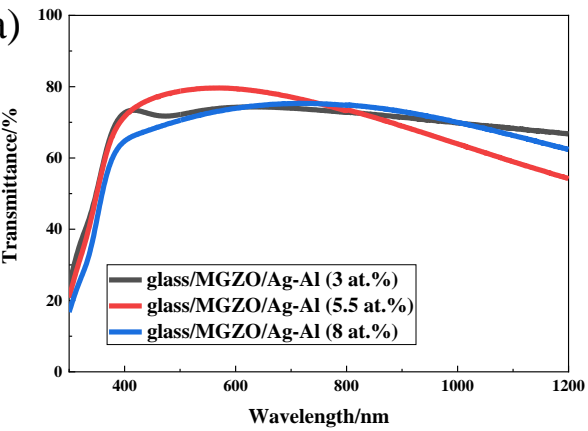

(b)

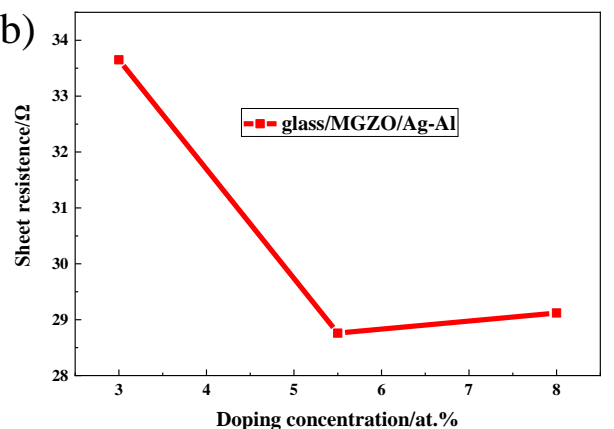

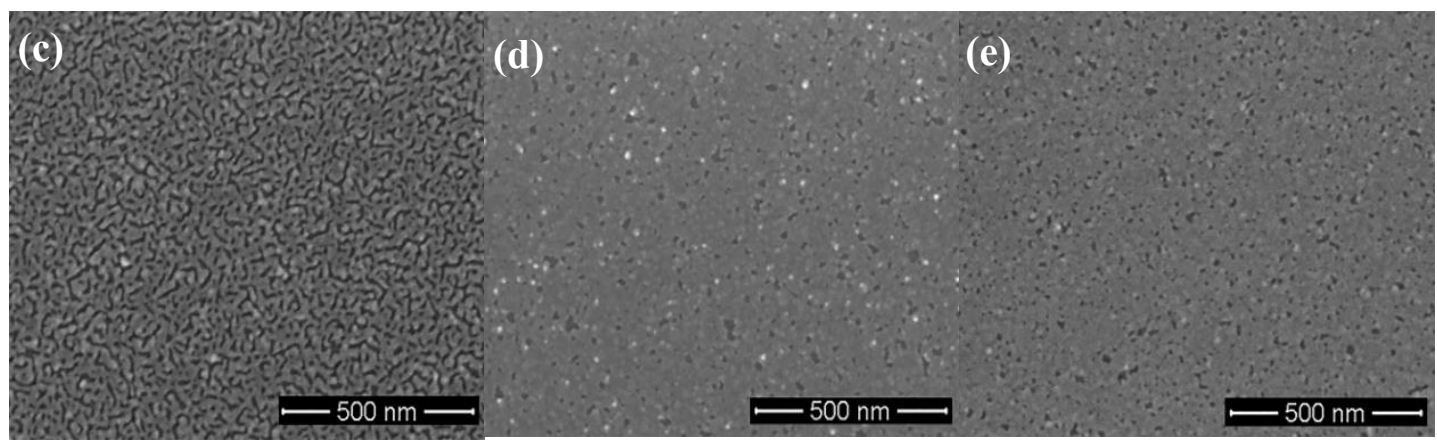

Figure S5. (a) Transmittance and (b) sheet resistance of glass/MGZO/7 nm Ag-Al films with different doping concentrations; SEM image of glass/MGZO/7 nm Ag-Al films with different doping concentrations: (c) 3 at.\%; (d) 5.5 at.\%; (e) 8 at.\%. Considering the optical performance, electrical performance and surface morphology, 5.5 at.\% is considered as the optimized $\mathrm{Al}$ doping concentration. 
III. Effect of different oxygen flow rate on performance of $4 \mathrm{~nm}$ thick $\operatorname{Ag}-\mathrm{Zn}(\mathrm{O})$ thin films.
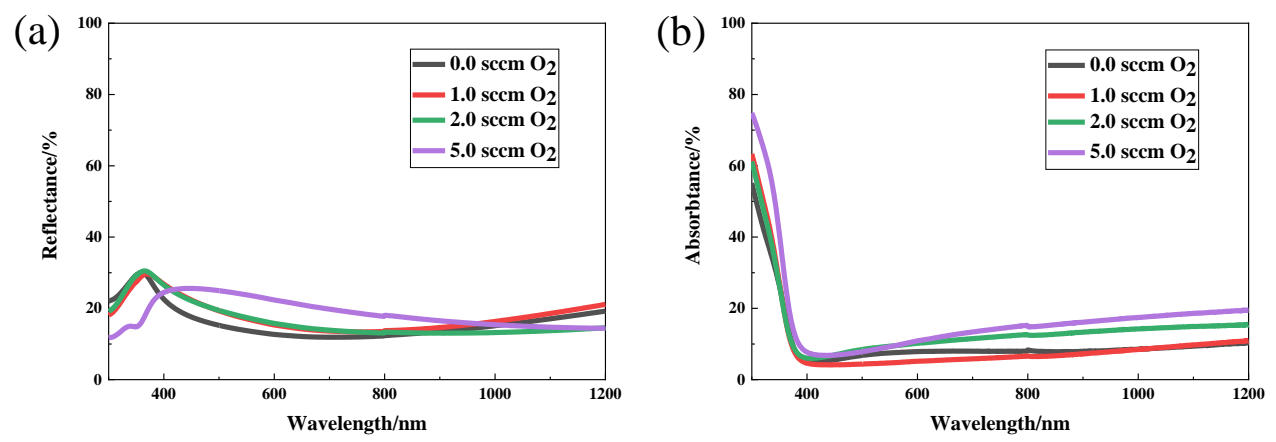

Figure S6. (a) Reflectance and (b) absorbance of glass/MGZO/Ag-Zn(O) films with different $\mathrm{O}_{2}$ flow. The reflectance increases with the increase of oxygen flow. The average absorbance has decreased from $8 \%$ to $6.8 \%(\lambda \sim 400-1200 \mathrm{~nm})$, when $1.0 \mathrm{sccm}$ $\mathrm{O}_{2}$ is introduced.
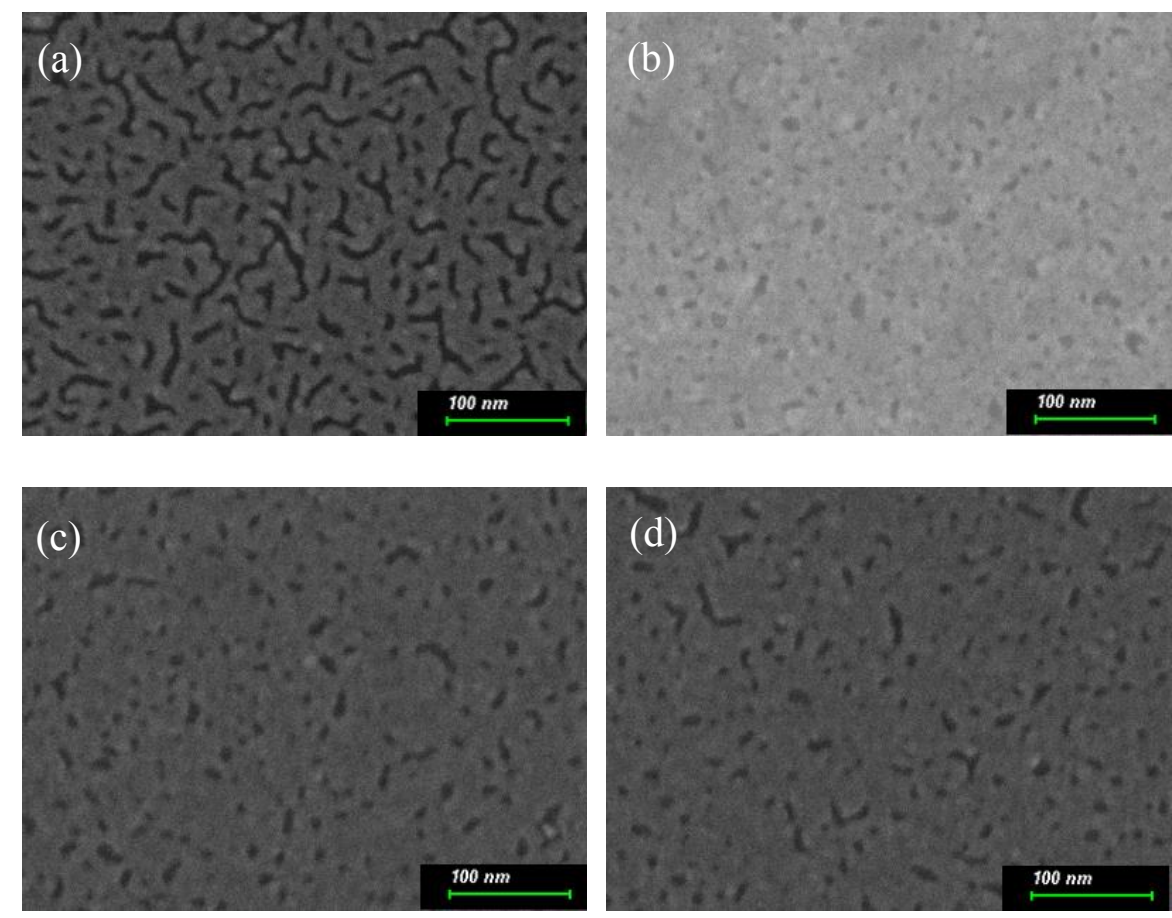

Figure S7. The surface morphology of glass/MGZO/Ag-Zn(O) with different $\mathrm{O}_{2}$ flow rates: (a) $0 \mathrm{sccm}$; (b) $1.0 \mathrm{sccm}$; (c) $2.0 \mathrm{sccm}$; (d) $5.0 \mathrm{sccm}$. The $4 \mathrm{~nm}$ thick $\mathrm{Ag}-\mathrm{Zn}(\mathrm{O})$ film transforms from a surface morphology with lots of unfilled gaps to a continuous surface. 


\section{Optical and electrical influences of trace $\mathrm{O}_{2}$ on the threshold thickness of $\operatorname{Ag}-\operatorname{Zn}(0)$ thin films.}
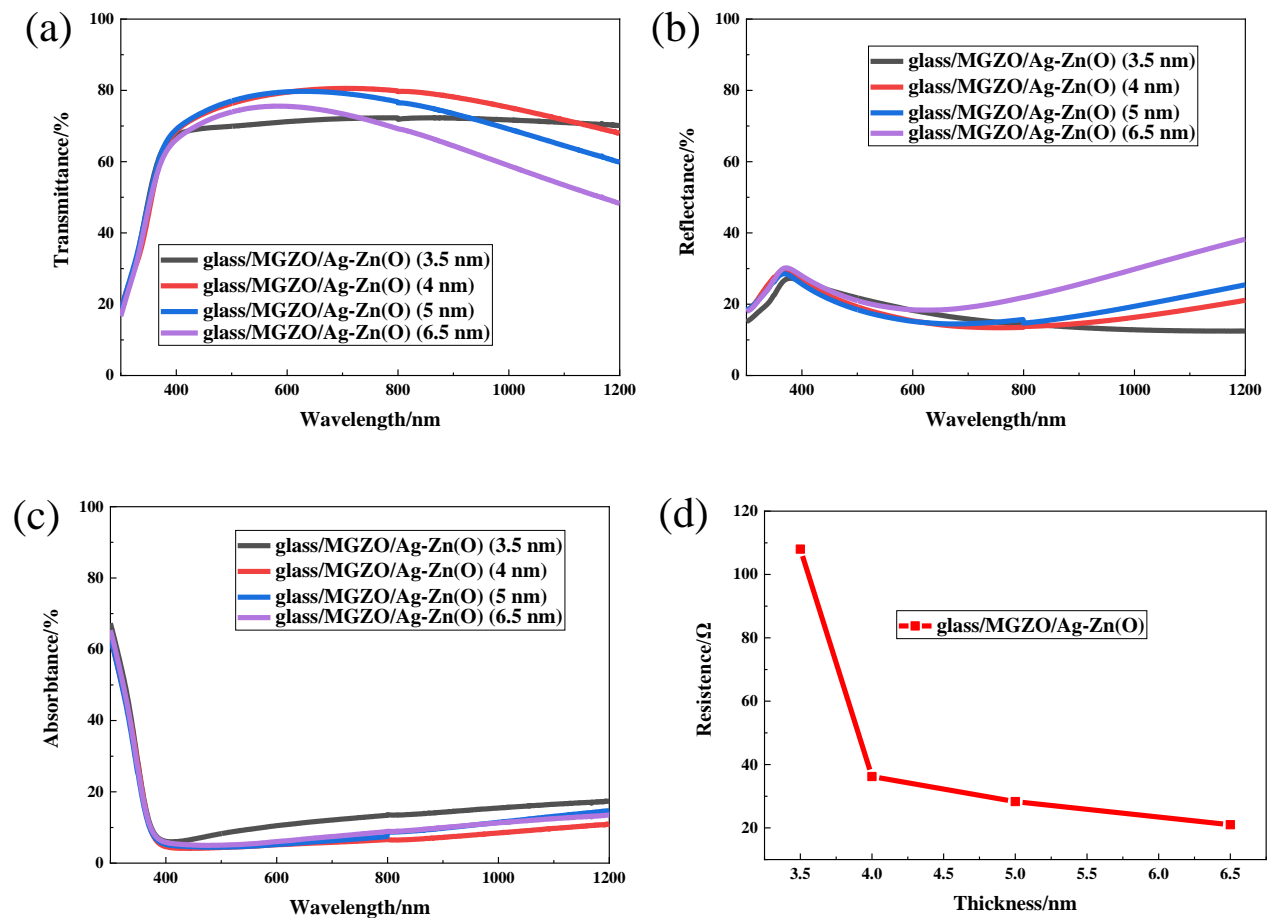

Figure S8. (a) Transmittance, (b) reflectance, (c) absorbance, (d) sheet resistance of glass/MGZO/Ag-Zn(O) with different thickness of $\operatorname{Ag}-\mathrm{Zn}(\mathrm{O})$ films (The $\mathrm{O}_{2}$ flow is fixed at $1.0 \mathrm{sccm})$. 


\section{Optical, electrical and structural performances of Ag-CdO thin films.}

(a)

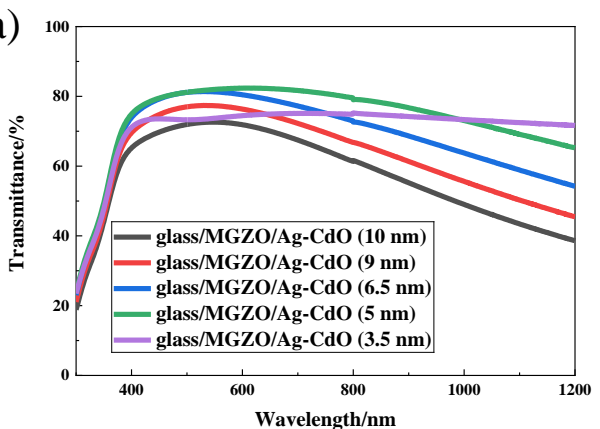

(c)

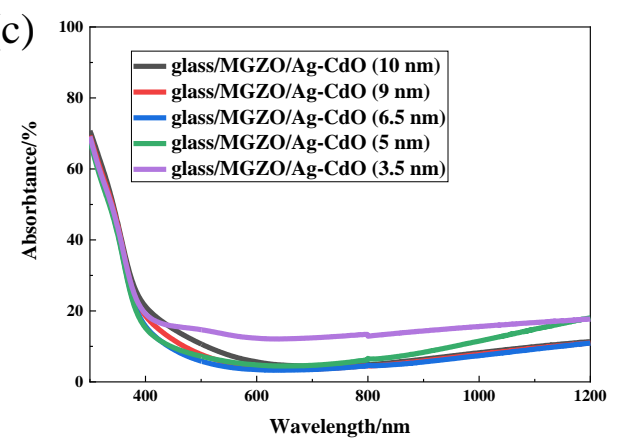

(b)

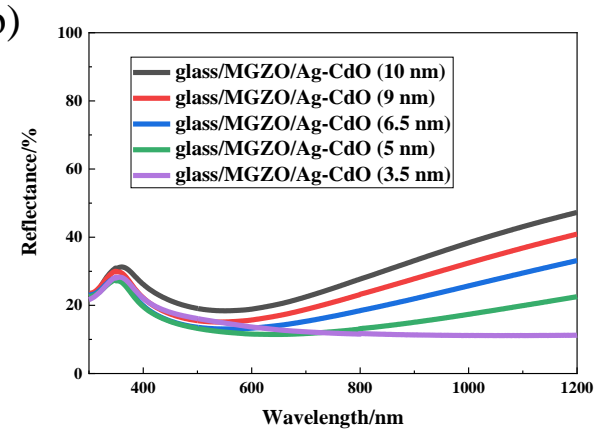

(d)

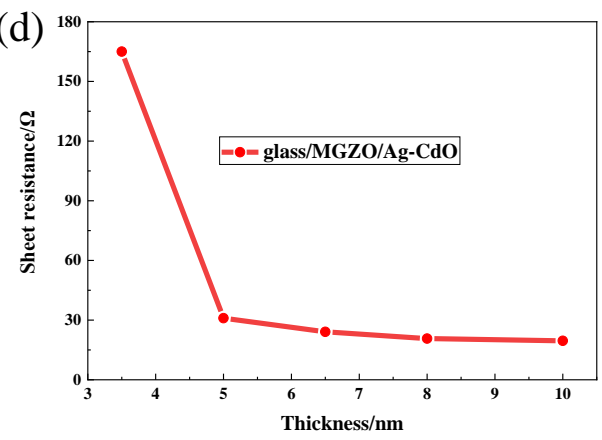

Figure S9. (a) transmittance, (b) reflectance, (c) absorbance and (d) sheet resistance of glass/MGZO/Ag-CdO with different thicknesses of Ag-CdO films.
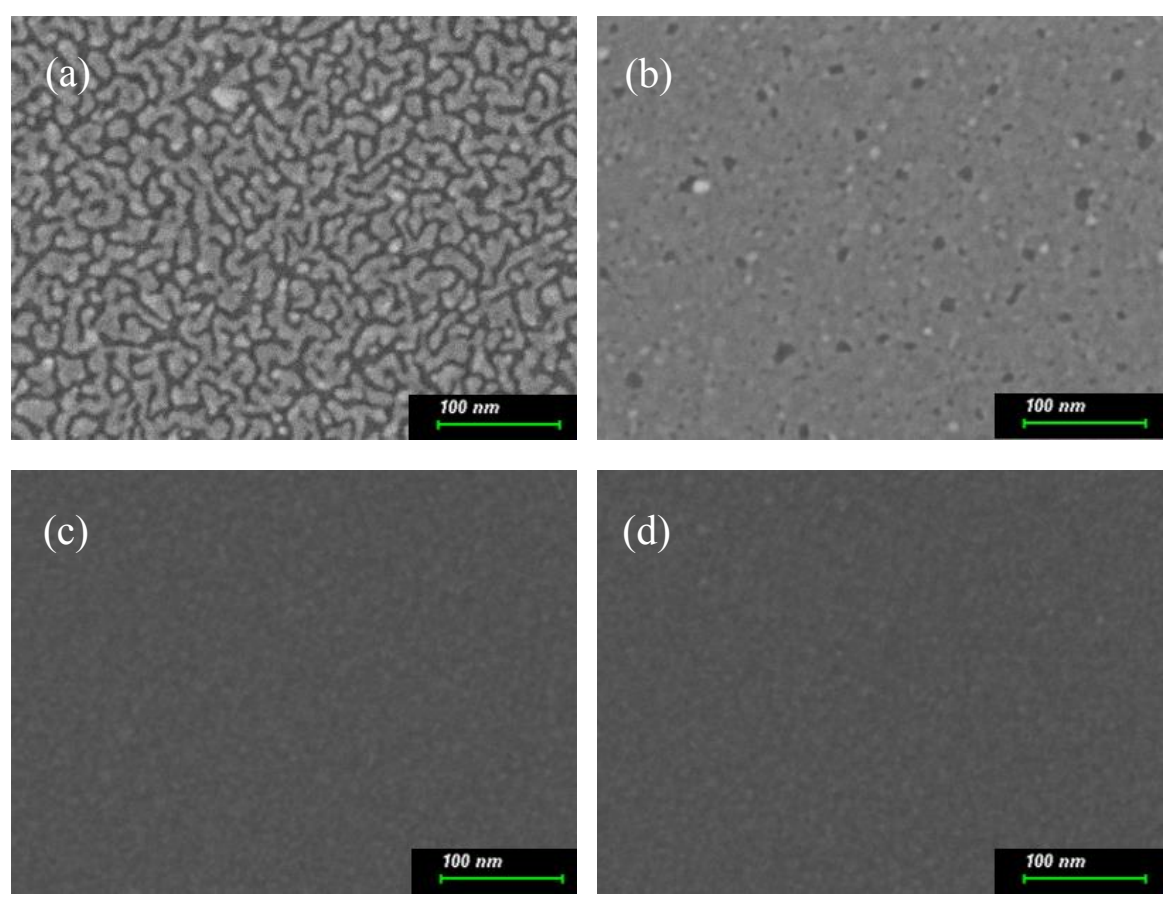

Figure S10. The surface morphology of glass/MGZO/Ag-CdO with different thicknesses of Ag-CdO films: (a) $3.5 \mathrm{~nm}$; (b) $5 \mathrm{~nm}$; (c) $6.5 \mathrm{~nm}$; (d) $8 \mathrm{~nm}$. 
VI. Performances of organic solar cells (OSCs) based on commercialized glass/ITO and glass/OMO (MGZO/Ag/MGZO) electrodes.
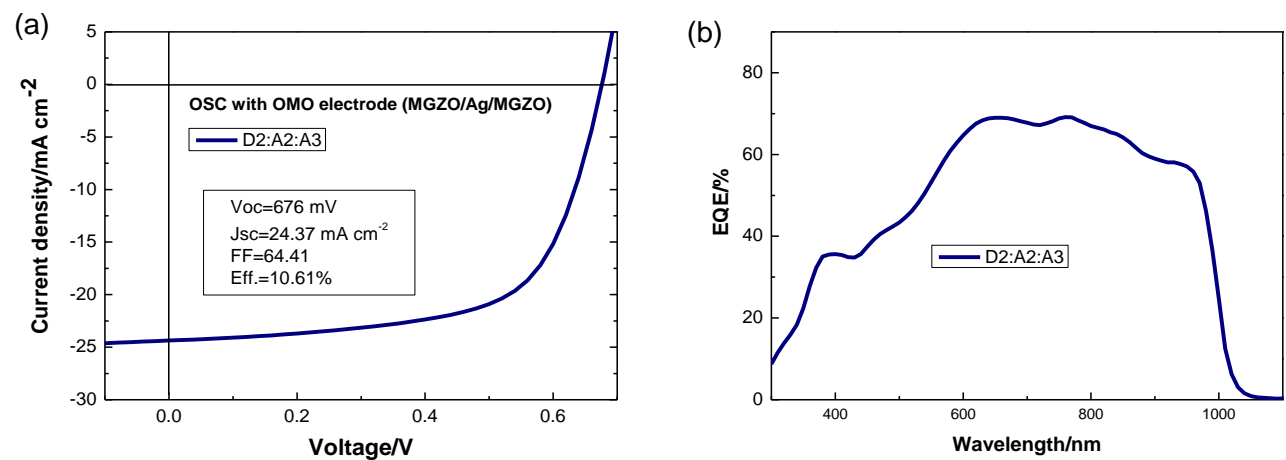

Figure S11. (a) $J-V$ curve and (b) EQE spectrum of OSC based on glass/OMO (MGZO/Ag/MGZO) electrode with the photoactive layer of D2:A2:A3.
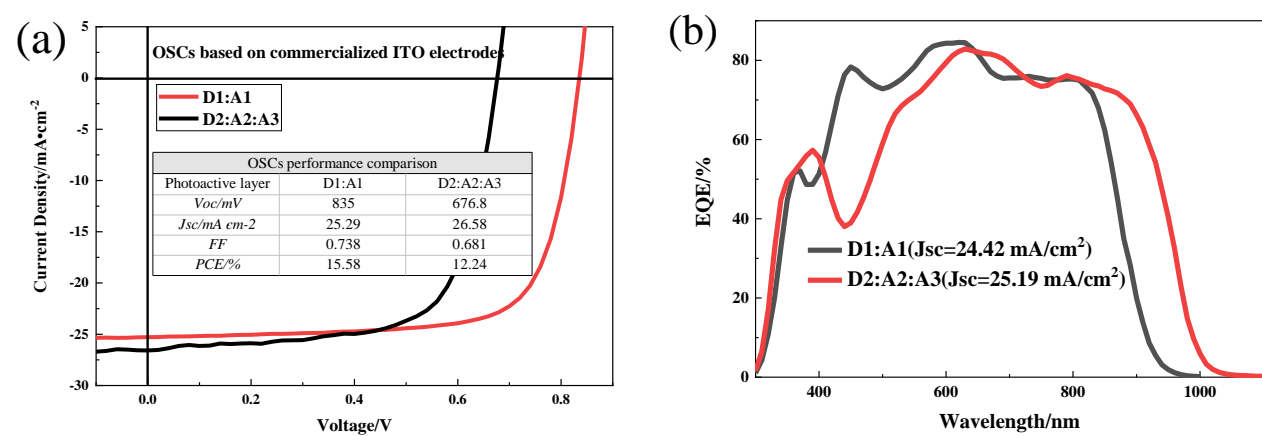

Figure S12. (a) $J-V$ characteristics and (b) EQE spectra of OSCs based on commercialized glass/ITO electrodes with different photoactive layers. 


\section{Table summary of typical OMO (oxide-metal-oxide) thin films.}

Table S1. Summary of optical and electrical properties of typical OMO compound films.

\begin{tabular}{|c|c|c|c|}
\hline OMO structure (nm) & Transmittance & $\begin{array}{c}\text { Sheet } \\
\text { resistance } \\
(\Omega / s q)\end{array}$ & Reference \\
\hline $\begin{array}{l}\mathrm{Ag}-\mathrm{Al} / \mathrm{ZnO} \\
\quad(7 / 40)\end{array}$ & $\begin{array}{c}92 \%(550 \mathrm{~nm}) \\
>80 \%(400-800 \mathrm{~nm})\end{array}$ & 23.4 & {$[1]$} \\
\hline $\begin{array}{l}\text { ITO/Ag-Cu/ITO } \\
(40 / 8 / 40)\end{array}$ & $\begin{aligned} & 98.5 \%(600 \mathrm{~nm}) \\
> & 96 \%(400-700 \mathrm{~nm})\end{aligned}$ & 12.6 & {$[2]$} \\
\hline $\begin{array}{c}\mathrm{ZnO} / \mathrm{Ag}-\mathrm{Cu} / \mathrm{Al}_{2} \mathrm{O}_{3} \\
(24 / 6.5 / 56)\end{array}$ & $100.3 \%(400-700)$ & 18.6 & {$[3]$} \\
\hline $\begin{array}{c}\mathrm{AZO} / \mathrm{Ag}_{-}-\mathrm{SnO}_{\mathrm{x}} / \mathrm{AZO} \\
(40 / 6 / 40)\end{array}$ & $79.1 \%(550 \mathrm{~nm})$ & 10.8 & {$[4]$} \\
\hline $\begin{array}{c}\mathrm{Al}_{2} \mathrm{O}_{3} / \mathrm{Ag} / \mathrm{MoO}_{\mathrm{x}} \\
(40 / 9 / 20)\end{array}$ & $93.85 \%(550 \mathrm{~nm})$ & 7.39 & {$[5]$} \\
\hline $\begin{array}{c}\mathrm{ZnO} / \mathrm{MUA} / \mathrm{Ag} / \mathrm{ZnO} \\
(40 / * / 10 / 20)\end{array}$ & $>80 \%(400-600 \mathrm{~nm})$ & 8.61 & {$[6]$} \\
\hline $\begin{array}{c}\mathrm{ZnO} / \mathrm{Cu}(\mathrm{N}) / \mathrm{ZnO} \\
(60 / 6.5 / 60)\end{array}$ & $84 \%(380-1000 \mathrm{~nm})$ & 20 & {$[7]$} \\
\hline $\begin{array}{c}\mathrm{MoO}_{3} / \mathrm{Au} / \mathrm{MoO}_{3} \\
(30 / 7 / 80)\end{array}$ & $\begin{array}{l}\sim 80 \%(500-700 \mathrm{~nm}) \\
74 \%(800-1100 \mathrm{~nm})\end{array}$ & 19.6 & {$[8]$} \\
\hline $\begin{array}{c}\mathrm{ZnO} / \mathrm{AgO}_{\mathrm{x}} / \mathrm{ZnO} \\
(50 / 6 / 50)\end{array}$ & $92 \%(400-1000 \mathrm{~nm})$ & 26.5 & [9] \\
\hline $\begin{array}{c}\mathrm{AZO} / \mathrm{Ag} / \mathrm{AZO} \\
(35 / 6 / 30)\end{array}$ & $93.6(500 \mathrm{~nm})$ & 5.6 & {$[10]$} \\
\hline $\begin{array}{c}\mathrm{SnO}_{\mathrm{x}} / \mathrm{Ag} / \mathrm{SnO}_{\mathrm{x}} \\
(20 / 7 / 20)\end{array}$ & $81 \%(390-780 \mathrm{~nm})$ & 10 & [11] \\
\hline $\begin{array}{c}\mathrm{MGZO} / \mathrm{Ag} / \mathrm{MGZO} \\
(40 / 9.5 / 45)\end{array}$ & $94.7 \%(400-800 \mathrm{~nm})$ & 10 & {$[12]$} \\
\hline $\begin{array}{c}\mathrm{ZnO} / \mathrm{AgO}_{\mathrm{x}} / \mathrm{Ag} / \mathrm{ZnO} \\
(5 / 1.5 / 6 / 25)\end{array}$ & $94 \%(400-800 \mathrm{~nm})$ & 12.5 & [13] \\
\hline
\end{tabular}


Table S2. Lattice strains and crystallite sizes of the 100-nm thick Ag-Zn(O) film with different $\mathrm{O}_{2}$ flow rates determined from the $\mathrm{Ag}$ (111) peak.

\begin{tabular}{|c|c|c|c|c|}
\hline $\mathrm{O}_{2}$ flow rate $/ \mathrm{sccm}$ & Peak position/2 $2 \theta$ & $\mathrm{FWHM} / 2 \theta$ & $\begin{array}{l}\text { Lattice } \\
\text { strain/\% }\end{array}$ & $\begin{array}{c}\text { Crystallite } \\
\text { size/nm }\end{array}$ \\
\hline 0 & 38.26 & 0.64 & 0.81 & 12.0 \\
\hline 1.0 & 38.06 & 0.81 & 1.02 & 10.2 \\
\hline 2.0 & 38.12 & 1.05 & 1.32 & 7.9 \\
\hline 5.0 & 38.16 & 1.56 & 1.97 & 5.3 \\
\hline
\end{tabular}

\section{References}

(1) Zhang, C.; Zhao, D.; Gu, D.; Kim, H.; Ling, T.; Wu, Y. K.; Guo, L. J. An Ultrathin, Smooth, and Low-loss Al-doped Ag Film and Its Application as a Transparent Electrode in Organic Photovoltaics. Adv. Mater. 2014, 26 (32), 5696-5701.

(2) Wang, H.; Ji, C.; Zhang, C.; Zhang, Y.; Zhang, Z.; Lu, Z.; Tan, J.; Guo, L. J. Highly Transparent and Broadband Electromagnetic Interference Shielding Based on Ultrathin Doped Ag and Conducting Oxides Hybrid Film Structures. ACS Appl. Mater. Inter. 2019, 11 (12), 11782-11791.

(3) Ji, C.; Liu, D.; Zhang, C.; Jay Guo, L. Ultrathin-Metal-Film-Based Transparent Electrodes with Relative Transmittance Surpassing 100. Nat. Commun. 2020, 11 (1), 3367.

(4) Wang, Z.; Li, J.; Xu, J.; Huang, J.; Yang, Y.; Tan, R.; Chen, G.; Fang, X.; Zhao, Y.; Song, W. Robust Ultrathin and Transparent AZO/Ag-SnO /AZO on Polyimide Substrate for Flexible Thin Film Heater with Temperature Over $400{ }^{\circ} \mathrm{C}$. J. Mater. Sci. Technol. 2020, 48, 156-162.

(5) Yang, X.; Gao, P.; Yang, Z.; Zhu, J.; Huang, F.; Ye, J. Optimizing Ultrathin Ag Films for High Performance Oxide-Metal-Oxide Flexible Transparent Electrodes through Surface Energy Modulation and Template-stripping Procedures. Sci. Rep. 2017, 7, 44576.

(6) Zou, J.; Li, C.; Chang, C.; Yip, H.; Jen A. Interfacial Engineering of Ultrathin Metal Film Transparent Electrode for Flexible Organic Photovoltaic Cells. Adv. Mater. 2014, 26, 3618-3623. 
(7) Zhao, G.; Kim, S. M.; Lee, S.-G.; Bae, T.-S.; Mun, C.; Lee, S.; Yu, H.; Lee, G.-H.; Lee, H.-S.; Song, M.; Yun, J. Bendable Solar Cells from Stable, Flexible, and Transparent Conducting Electrodes Fabricated Using a Nitrogen-doped Ultrathin Copper Film. Adv. Funct. Mater. 2016, 26 (23), 4180-4191.

(8) Wang, Z.; Zhu, X.; Zuo, S.; Chen, M.; Zhang, C.; Wang, C.; Ren, X.; Yang, Z.; Liu, Z.; Xu, X.; Chang, Q.; Yang, S.; Meng, F.; Liu, Z.; Yuan, N.; Ding, J.; Liu, S.; Yang, D. 27\%-efficiency Four-terminal Perovskite/silicon Tandem Solar Cells by Sandwiched Gold Nanomesh. Adv. Funct. Mater. 2019, 1908298.

(9) Wang, W.; Song, M.; Bae, T.-S.; Park, Y. H.; Kang, Y.-C.; Lee, S.-G.; Kim, S.-Y.; Kim, D. H.; Lee, S.; Min, G.; Lee, G.-H.; Kang, J.-W.; Yun, J. Transparent Ultrathin Oxygen-doped Silver Electrodes for Flexible Organic Solar Cells. Adv. Funct. Mater. 2014, 24 (11), 1551-1561.

(10) Li, P.; Li, H.; Ma, J.; Zhou, Y.; Zhang, W.; Cong, L.; Xu, H.; Liu, Y. Structural Optimization of Oxide/Metal/Oxide Transparent Conductors for High-performance Low-emissivity Heaters. Adv. Mater. Inter. 2018, 5 (24), 1801287.

(11) Zhao, J.; Brinkmann, K.; Hu, T.; Pourdavoud, N.; Becker, T.; Gahlmann, T.; Heiderhoff, R.; Polywka, A.; Görrn, P.; Chen, Y.; Cheng, B.; Riedl, T. Self-encapsulating Thermostable and Air-resilient Semitransparent Perovskite Solar Cells. Adv. Energy Mater. 2017, 7, 1602599.

(12) Zhang, Y.; Liu, Z.; Ji, C.; Chen, X.; Hou, G.; Li, Y.; Zhou, X.; Cui, X.; Yang, X.; Ren, C.; Liu, D.; Guo, L. J.; Zhao, Y.; Zhang, X. Low-temperature Oxide/Metal/Oxide Multilayer Films as Highly Transparent Conductive Electrodes for Optoelectronic Devices. ACS Appl. Energy Mater. 2021, 4(7), 6553-6561.

(13) Zhao, G.; Shen, W.; Jeong, E.; Lee, S.G.; Yu, S.M.; Bae, T.S.; Lee, G.H.; Han, S.Z.; Tang, J.; Choi, E.A.; Yun, J. Ultrathin Silver Film Electrodes with Ultralow Optical and Electrical Losses for Flexible Organic Photovoltaics. ACS Appl. Mater. Inter. 2018, 10, 27510-27520. 